\title{
Aspect énergétique du relèvement par osmose inverse du degré alcoométrique des piquettes à distiller
}

\author{
par \\ R. AUDINOS*, M. CABRIT ${ }^{* *}$ et M. MOUTOUNET**
}

\section{R é s u m é}

La concentration des piquettes par osmose inverse préalablement à leur distillation est possible avec les membranes actuellement disponibles. Le coût énergétique de l'opération est comparé avec celui de la distillation simple et avec celui de la distillation avec recompression des vapeurs. Pour des membranes de performances données (IRCHA), l'influence du taux de recyclage et de la surface de membrane a été mise en évidence.

\section{S u m m a r y}

ENERGETIC CONSIDERATION ABOUT THE CONCENTRATION WITH REVERSE OSMOSIS OF «PIQUETTES» BEFORE DISTILLATION

It is possible to concentrate "piquettes" by reverse osmosis, before the distillation of these alcoholic solutions issued from wine production. Some membranes are available. However, it is necessary to compare the energy consumption of this preconcentration step, to the energy necessary used in conventional distillation or in distillation with vapor recompression. For the used membranes (IRCHA) the influence of the recirculation rate and the membrane area are studied.

* Laboratoire de chimie physique et électrochimie, Université Paul-Sabatier, 118, route de Narbonne - 31400 Toulouse.

** Laboratoire de technologie des produits végétaux, I.N.R.A., chemin de Borde Rouge Auzeville - 31320 Castanet-Tolosan. 


\section{INTRODUCTION}

Il est couramment admis que l'alcool obtenu à partir des ressources agricoles est l'une des possibilités existantes pour subvenir aux besoins en carburants liquides (Grégor, Seffries, 1979). Or les viticulteurs commercialisant leur récolte sont assujettis à l'obligation de prestations viniques : c'est une taxe dont ils sont redevables à l'Etat. Les prestations viniques ont été rendues olbigatoires par le décret loi du 12 juillet 1937 dans le but d'assainir qualitativement le marché des vins. Le législateur impose la fourniture d'alcool à l'Etat correspondant à $10 \%$ de la récolte exprimée en alcool pur sur la base du degré minimum des vins de pays dans la région viticole considérée. L'alcool nécessaire à assurer les prestations viniques est récupéré, dans la plupart des cas par la distillation de piquettes. Celles-ci sont des solutions à bas degré alcoométrique obtenues lors de la vinification. A l'issue du pressurage, l'alcool contenu dans les marcs est extrait par une percolation à l'eau (fig. 1). Dans le cas de la vinification en blanc, où le pressurage est réalisé avant la fermentation, il est procédé à un levurage lors de l'apport d'eau : en effet les marcs doux contiennent une quantité de sucre fermentescible non négligeable. Il en est de même lors de la production de vins par thermo-vinification.

Le liquide obtenu à l'issue de la percolation est la piquette dont la teneur en alcool est comprise, en général, entre 3 et $5 \%$ en volume.

Si antérieurement la distillation uniquement thermique était une opération rentable, deux facteurs indépendants imposent de recourir à un enrichissement préalable :

- d'une part, l'augmentation du prix de l'énergie thermique conduit à rechercher une meilleure utilisation de celle-ci : c'est ainsi que se sont développés l'emploi des évaporateurs à multiple effet ou l'usage de la recompression mécanique des vapeurs;

- d'autre part, l'amélioration des traitements préalables à la vinification, notamment par l'emploi de pressoirs horizontaux, fait que la quantité d'alcool extractible par tonne de marc décroît (Cabrit, 1982).

Parmi l'arsenal des méthodes disponibles, il nous est apparu que les techniques à membranes pouvaient se révéler intéressantes pour effectuer une concentration préalable à la distillation des piquettes. En effet, en plus de celles-ci (Mafart, 1981), les diverses méthodes de séparation du mélange eautalcool peuvent être rassemblées en quatre groupes :

- celles faisant appel à des déshydratants solides, qu'ils soient de nature organique comme l'amidon, le blé concassé, ou minérale comme les zéolithes et qui adsorbent l'eau; 


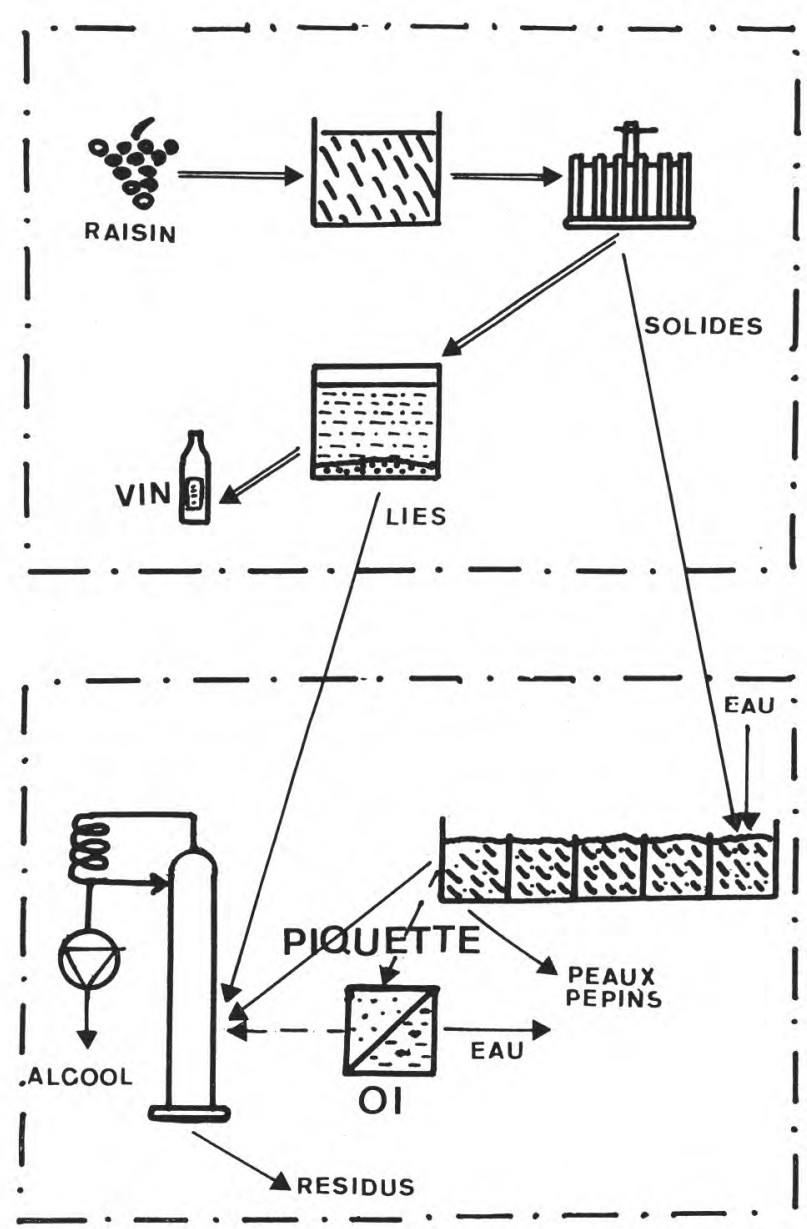

fig. 1

Schéma du principe d'obtention des piquettes.

Process flow diagram for "piquette » production.

- celles reposant sur l'emploi de solvants immiscibles à l'eau comme le dibutylphtalate, qui extraient l'alcool en phase liquide;

- celles utilisant un abaissement de température pour provoquer la congélation de l'un des constituants, compte tenu de l'existence à $-123^{\circ} \mathrm{C}$ d'un eutectique titrant $92 \%$;

- celles ayant recours à un fluide à l'état hypercritique, comme le dioxyde de carbone, qui entraîne l'alcool à l'état de gaz. 
La séparation du mélange eau-alcool par une technique à membrane, donc sans avoir recours à un changement d'état physique, conduit à obtenir deux solutions : l'une riche, l'autre pauvre. L'écart $\Delta \pi$ de pression osmotique qui existe alors entre les deux faces de la membrane ne permet d'envisager l'application d'une technique à membrane qu'aux deux extrémités de l'échelle des concentrations. En effet, en admettant que les membranes d'osmose inverse actuellement disponibles puissent supporter sans dommage une différence de pression de l'ordre de 100 bars, (10 M Pa), le calcul de l'équilibre thermodynamique, qui peut se traduire par la relation de Van't Hoff, montre que la concentration de la substance la moins abondante ne saurait dépasser $4 \mathrm{kmol} / \mathrm{m}^{3}$ environ. Par suite, l'osmose inverse ne peut être utilisée pour concentrer en alcool des solutions aqueuses qu'entre 0 et $20 \%$ d'une part ou qu'entre 95 et $100 \%$ d'autre part. Toutefois la sélectivité des membranes ne doit pas être la même selon la zone de concentration considérée : dans le cas de solutions à bas degré alcoométrique, la membrane semi-perméable doit arrêter l'alcool et laisser passer l'eau, tandis que pour les mélanges à fort degré, la membrane doit être perméable à l'alcool et imperméable à l'eau.

Les membranes disponibles sur le marché actuellement ont été conçues surtout pour déminéraliser des eaux salines : par suite, elles ont toujours une forte perméabilité à l'eau et ne peuvent donc être utilisées que pour concentrer les solutions hydroalcooliques diluées. Cependant, leur pouvoir de sélection est surtout manifeste vis-à-vis des systèmes eau-chlorure de sodium. Par contre, leur emploi pour retenir l'alcool conduit à des résultats assez aléatoires. Aussi, est-il nécessaire de procéder à des essais systématiques des diverses membranes disponibles dans des conditions proches de celle de l'utilisation envisagée.

La figure 2 montre que sur le marché nord-américain les membranes TFC et FT ont, sous 60 bars et pour des solutions aqueuses d'alcool à $6 \%$ en volume, le meilleur taux de rejet global TRG, bien que celui-ci ne dépasse pas $60 \%$. Cependant, leur coefficient de performance $\mathrm{k}_{\mathrm{T}}$ à $25^{\circ} \mathrm{C}$ peut varier du simple au double $(\mathrm{FT})$, tout en restant inférieur à $13 \mathrm{~kg} / \mathrm{j}-\mathrm{m}^{2}$ bar $\left(1,5 \mathrm{~g} / \mathrm{s} \mathrm{m}^{2} \mathrm{~Pa}\right)$. Par contre, d'autres membranes, telle la PEI, sont moins sélectives, mais plus perméables (Mehta, 1982).

Néanmoins, ces résultats montrent bien qu'il n'est pas impossible de concentrer les solutions hydroalcooliques, dans la mesure où il est permis d'utiliser des membranes performantes. Aussi dans le travail qui suit, avons-nous monté des membranes récemment mises au point pour étudier l'économie d'énergie que peut apporter une concentration par osmose inverse, préalablement à la distillation des piquettes. 


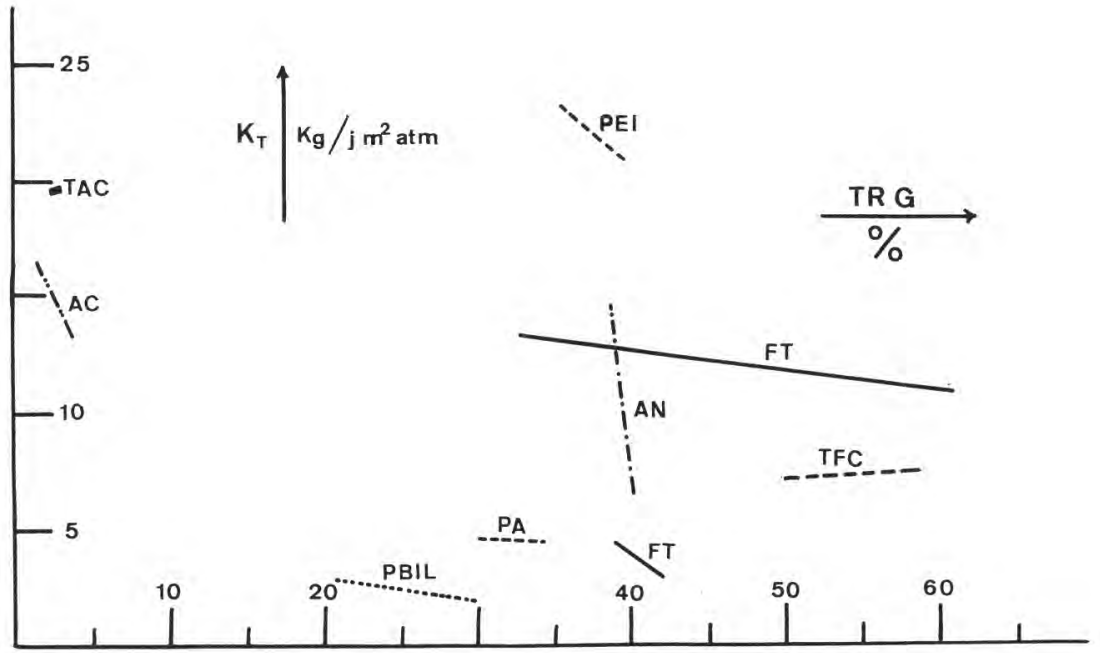

fig. 2

Relation entre le coeefficient de performance $\mathrm{k}_{\mathrm{T}}$ et le taux de rejet TRG $=1-\left(\mathrm{C}_{\mathrm{P}} / \mathrm{C}^{\circ}{ }_{\mathrm{R}}\right)$ pour les membranes d'osmose inverse commercialisée (d'après MeHTA D., 1981)

AN : acylonitrile (Sumitomo) ; AC : acétate de cellulose (Universal Oil Products) ; FT : composition non dévoilée (Filmtec) ; PA : polyamide (Dupont) ; PEI : polyéthylène imine (Universal Oil Products) ; PBIL : polybenzimidazalone (Abcor, Teijin) ; TAC : triacélate et acétate de cellulose (Universal Oil Products) ; TFC : composition non dévoilée (Universal Oil Products).

Relation between the performance coefficient $k_{T}$ and the ethanol rejection TRG $=1-\left(C_{P} / C^{\circ}{ }_{R}\right)$ for some available membranes $A N$ : acrylonitrile polyester; AC: acetate cellulose; FT: undisclosed composition; PA: polyamide; PEI: polyethylene imine; PBIL: polybenzimidazalone; TAC: cellulose acetate and triacetate blend; TFC: undisclosed composition.

\section{CONDITIONS EXPERIMENTALES}

\subsection{Les membranes}

Les membranes composites utilisées, confectionnées par l'IRCHA, ont une peau microporeuse de polyamide méthylolé, déposée sur un support mésoporeux de polysulfone.

Les performances de ces membranes ont d'abord été contrôlées avec un mélange synthétique eau-éthanol à $2 \%$, à $25^{\circ} \mathrm{C}$, sous une pression de 60 bars, après un compactage à 80 bars. Les valeurs obtenues pour le coefficient de performance et le taux de rejet observé, $\mathrm{TR}=1-\mathrm{C}_{\mathrm{P}} / \mathrm{C}_{\mathrm{R}}$ avec notre montage corespondent à celles fournies par le fabricant (fig. 3). 


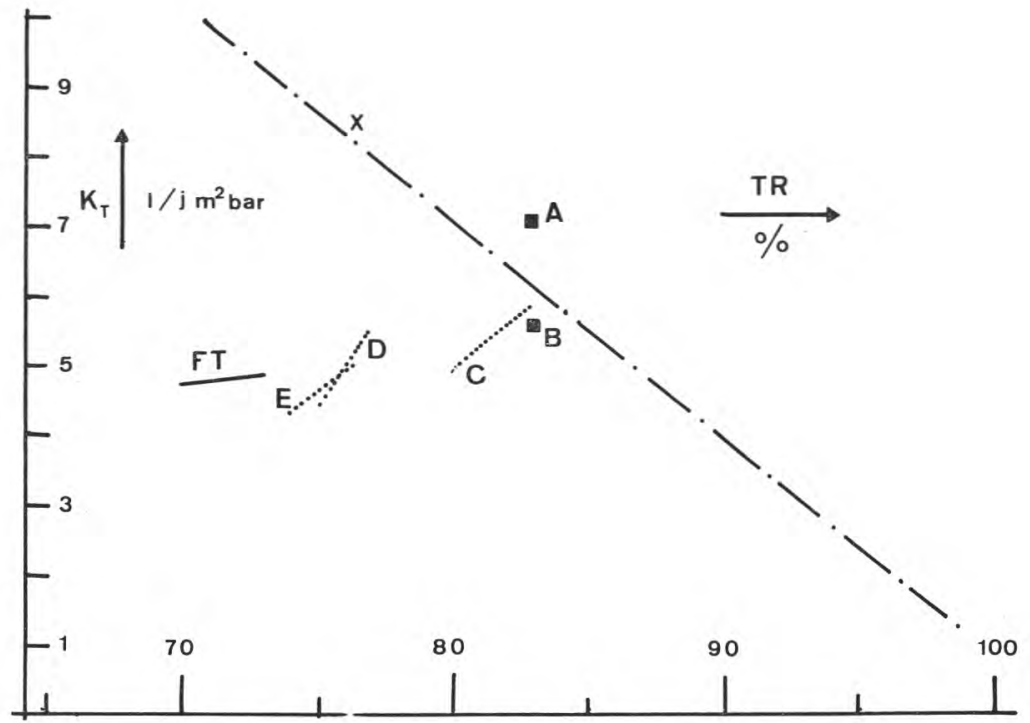

fig. 3

Exemple de droite reliant le coefficient de performance $\mathrm{k}_{\mathrm{T}}$ et le taux de rejet TR $=1-\left(\mathrm{C}_{\mathrm{P}} / \mathrm{C}_{\mathrm{R}}\right)$ pour des membranes expérimentales $\mathrm{X}$. Membranes utilisées A, B, C, D, E (Ircha) et FT (Filmtec).

A linear relation between the performance coefficient $k_{T}$ and the ethanol rejection $T R=1\left(C_{P} / C_{R}\right)$ for some experimental membranes $X$. $A, B, C, D$, E: Ircha membranes; FT: Filmtec membranes.

\subsection{Le montage}

Le montage utilisé comporte quatre modules de laboratoire d'osmose inverse de $300 \mathrm{~cm}^{2}$ de surface utile. Le rétentat est recyclé en permanence jusqu'à l'obtention du degré alcoométrique désiré, tandis que le perméat est continuellement évacué (fig. 4). La vitesse de passage dans les modules peut être modifiée en faisant varier le débit volumétrique de la pompe. De plus, pour éviter le développement de bactéries acétiques, le bac du rétentat est placé sous un très léger balayage d'azote.

\subsection{La pression}

Le choix de la pression de 60 bars a été dicté par le souci de disposer d'une pression effective suffisante, tout au long de l'opération, même en présence d'acide tartrique. Ainsi, dans le cas d'une piquette contenant en volume $2 \%$ d'éthanol, la pression osmotique peut être estimée à 10 bars. Il est possible, toutes choses égales par ailleurs, de la concentrer au maximum six fois. Pratiquement, 


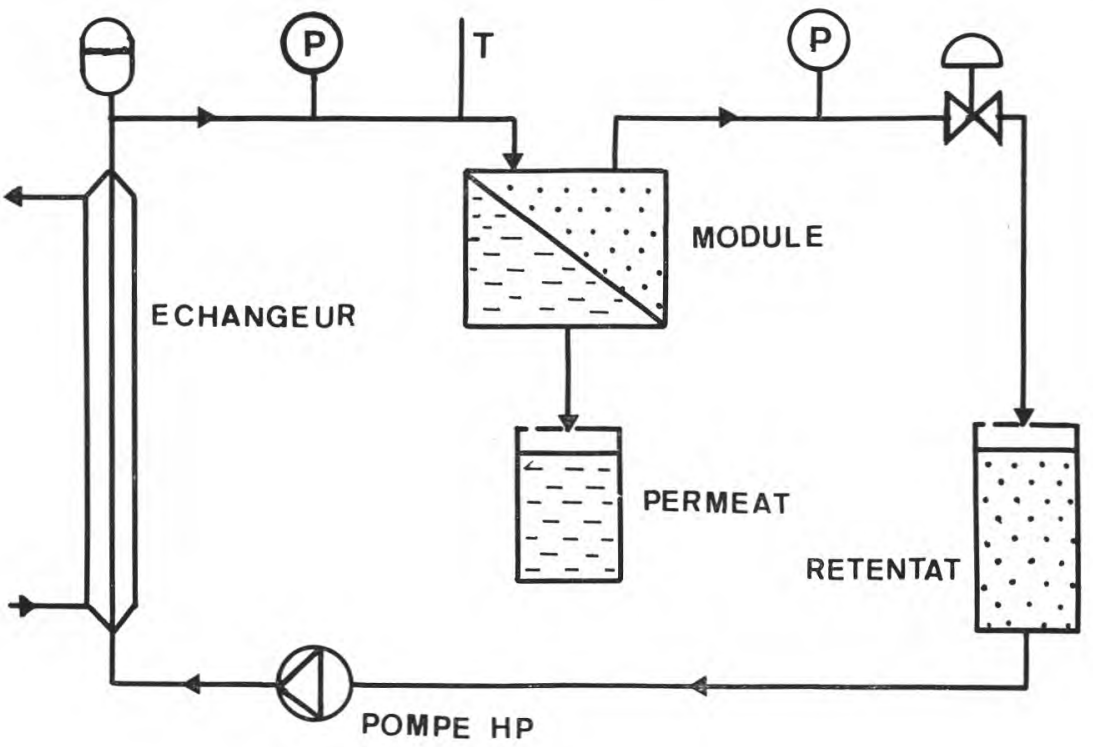

fig. 4

Schéma du montage.

Process flow diagram for reverse osmosis.

le facteur de concentration $\mathrm{FC}=\mathrm{C}_{\mathrm{T}} / \mathrm{C}^{\circ}{ }_{\mathrm{R}}$ adopté est voisin de 3 , ce qui permet d'obtenir en fin d'opération, une pression effective de l'ordre de 30 bars, en supposant que l'osmosat n'est constitué que par de l'eau.

En effet, les essais réalisés avec un mélange synthétique contenant en plus de l'éthanol $2 \mathrm{~g}$ d'acide tartrique par litre, montrent que le degré alcoométrique du rétentat n'augmente plus par suite de la cristallisation du tartrate acide de potassium. Toutefois ces précipitations peuvent être évitées par un traitement soit par le froid, soit par échange d'ions, soit par électrodialyse (Moutonnet, Lurton, Audinos, 1981).

\subsection{Les conditions opératoires}

Compte tenu des résultats obtenus avec les solutions synthétiques, la concentration des piquettes de teneur initiale proche de $4 \%$ a été effectuée en recyclant totalement le rétentat et en appliquant une pression de 60 bars, la température étant régulée à $25^{\circ} \mathrm{C}$. Une fois ces trois variables fixées, seuls deux paramètres primordiaux concernant le transfert de matière transmembranaire dans le module ont été modifiés : 
d'énergie ;

- d'une part, la vitesse de circulation dont dépend l'apport

- d'autre part, les caractéristiques de la membrane elle-même.

L'ensemble des conditions opératoires est résumé dans le tableau 1.

\section{TABLEAU $1-T A B L E 1$}

Conditions opératoires. $\mathrm{T}=298 \mathrm{~K}, \Delta \mathrm{P}=60$ bars

* pour une solution hydroalcoolique à $2 \%$ en volume

Operating conditions. $T=298 K, \Delta P=60$ bars

* with an aqueous solution of ethanol, $2 \%$

\begin{tabular}{|c|c|c|c|c|c|c|c|c|}
\hline Essai & $\begin{array}{l}\text { Mem- } \\
\text { brane }\end{array}$ & $\mathrm{TR}^{*}$ & $\mathrm{~K}^{*_{T}}$ & $\stackrel{\circ}{\mathrm{V}}_{\mathrm{c}}$ & $\mathrm{u}$ & $\mathrm{t}$ & $\mathrm{V}_{\mathrm{R}}^{\mathrm{o}}$ & $\mathrm{C}^{\circ}{ }_{\mathrm{R}}$ \\
\hline $\mathrm{n}^{\circ}$ & code & $\%$ & $1 / \mathrm{jm}^{2} \mathrm{bar}$ & $1 / \mathrm{h}$ & $\mathrm{cm} / \mathrm{sec}$ & heure & 1 & $\%$ vol \\
\hline 1 & A & 83 & 7,20 & 26,8 & 9,4 & 71 & 10 & 4,14 \\
\hline 2 & B & 83 & 5,64 & 69,5 & 24,3 & 143 & 15 & 3,84 \\
\hline 3 & C & 83 & 7,20 & 69,5 & 24,3 & 73 & 15 & 3,95 \\
\hline
\end{tabular}

\section{LES RESULTATS EXPERIMENTAUX}

\subsection{Les résultats analytiques}

Les piquettes ne sauraient être assimilées à de simples solutions hydroalcooliques. Leur analyse, avant et après traitement, fait clairement apparaître que le comportement des membranes utilisées n'est pas identique vis-à-vis de toutes les substances présentes. Le tableau 2 est un exemple des résultats analytiques obtenus. Il indique que dans les conditions expérimentales, ce type de membrane semiperméable présente une perméabilité à l'éthanol, alors qu'elle est totalement imperméable à l'acide tartrique et aux matières colorantes. Toutefois, le facteur de concentration, proche de 1,5 pour l'éthanol et l'acide acétique, est deux fois plus important pour le glycérol et les anthocyanes.

\subsection{Les bilans matière}

Les grandeurs mesurées, en plus des variables fixées, ont été les volumes et les concentrations. Les valeurs brutes font apparaître que contrairement à la solution synthétique éthanol-acide tartrique, 


\section{$T A B L E A U 2-T A B L E 2$}

Composition chimique d'une piquette, du rétentat et de l'osmosat correspondant

Analytical composition of a «piquette», of a concentrate, of a permeate

Densité $20 / 20$

Degré à $20^{\circ} \mathrm{C}$

Sucres $\mathrm{g} / \mathrm{l}$

Glycérol g/l

Azote total $\mathrm{mg} / \mathrm{l}$

Acidité totale $\mathrm{gH}_{2} \mathrm{SO}_{4} / 1$

Acidité volatile $\mathrm{gH}_{2} \mathrm{SO}_{4} / 1$

Acide tartrique méq/1

Acide malique en méq/1

$\mathrm{pH}$

$\mathrm{K}$ (après passage sur résines) en méq/1

\begin{tabular}{|c|c|c|}
\hline Piquette & Concentrat & Perméat \\
\hline 1,00981 & 1,03726 & 0,99839 \\
\hline 4,14 & 6,29 & 2,31 \\
\hline 2,54 & 6,07 & 0,23 \\
\hline 3,90 & 11,41 & 0,56 \\
\hline 635 & 1654 & 33 \\
\hline 8,30 & 20,60 & 2,95 \\
\hline 4,22 & 8,50 & 2,59 \\
\hline 15.8 & 19,2 & 0 \\
\hline 0 & 0 & 0 \\
\hline 3,82 & 3,90 & 3,63 \\
\hline 59,20 & 110,2 & 9,20 \\
\hline 0,60 & 1,82 & 0,17 \\
\hline 12,00 & 36,00 & 0,65 \\
\hline 16,6 & 54,1 & 0,8 \\
\hline 8,2 & 8,9 & traces \\
\hline 1,34 & 8,3 & traces \\
\hline 6 & 9 & \\
\hline 12 & 16 & 0 \\
\hline 0,144 & 0,654 & 0 \\
\hline 0,184 & 0,723 & 0 \\
\hline 1,983 & 5,871 & 0,048 \\
\hline 0,298 & 0,668 & 0,002 \\
\hline 0,775 & 2,756 & 0 \\
\hline
\end{tabular}

une teneur en alcool supérieure à $6 \%$ peut être facilement obtenue. L'évolution des concentrations au cours du temps, montre qu'un tel titre peut être dépassé (fig. 5). Cependant l'établissement des bilans matière impose de rectifier ces valeurs brutes. En effet, le bilan global permet bien de retrouver la totalité des volumes mis en jeu, compte tenu d'une fuite inhérente au type de pompe utilisée. Par contre, le bilan en éthanol fait apparaître une perte non négligeable, imputable uniquement à une évaporation partielle à partir des bacs de réception.

Par suite, les valeurs brutes, confirmées par deux analyses distinctes, ont été corrigées en supposant un taux d'évaporation identique pour toutes les solutions. L'ensemble de ces résultats est rassemblé dans le tableau 3. Il montre, toutes choses égales par ailleurs, que la concentration finale du rétentat augmente peu avec la vitesse de circulation (essais 1 et 3 ). Par contre, l'emploi d'une 

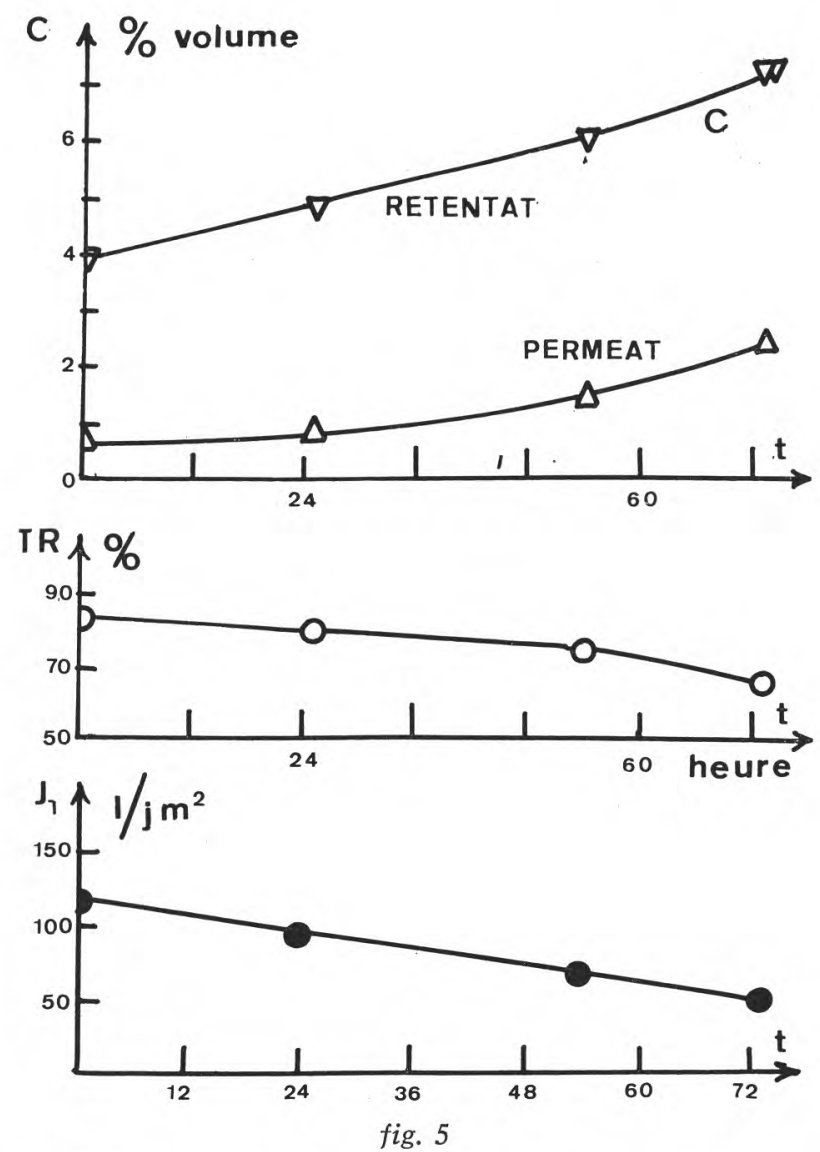

Evolution des principaux paramètres. Cas de l'essai $\mathrm{n}^{\circ} 3$ $\mathrm{a}$ : concentration du rétentat et du perméat ; b : taux de rejet ; c : densité du flux du perméat.

Variation with time of some parameters. Experiment 3 $a$ : permeate and concentrate concentrations; $b$ : rejection; c: permeate flux.

membrane moins perméable (essai 2) se révèle moins efficace pour obtenir la teneur désirée dans le rétentat, mais moins dispendieuse quant à la quantité d'éthanol emportée par le perméat, en principe en pure perte.

\subsection{La perméabilité et la sélectivité}

La mesure instantanée des débits permet de suivre l'évolution des densités des flux transmembranaires $\mathrm{J}_{\text {T. }}$ Ceux-ci décroissent au 


\section{TABLEAU $3-T A B L E 3$ \\ Bilans matière}

Material balances

\begin{tabular}{|c|c|c|c|c|c|c|c|c|c|}
\hline Essai & $\mathrm{Vt}_{\mathrm{R}}$ & $\mathrm{Cf}_{\mathrm{R}}$ & $\mathrm{Cf}_{R \mathrm{~F}}$ & $\mathrm{~V}_{\mathrm{P}}$ & $\mathrm{Cf}_{\mathrm{P}}$ & $\mathrm{Cf}_{\mathrm{PF}}$ & $\mathrm{Vf}_{\mathrm{F}}$ & $\mathrm{Cf}_{\mathrm{F}}$ & $\mathrm{Cf}_{\mathrm{FF}}$ \\
\hline $\mathrm{n}^{\circ}$ & 1 & $\%$ vol & $\%$ vol & 1 & $\%$ vol & $\%$ vol & 1 & $\%$ vol & $\%$ vol \\
\hline 1 & 2,62 & 6,29 & 7,20 & 6,62 & 2,31 & 2,64 & 0,76 & 12,67 & 7,20 \\
\hline 2 & 7,80 & 5,57 & 5,97 & 5,96 & 0,56 & 0,60 & 1,24 & 8,72 & 5,97 \\
\hline 3 & 5,70 & 7,52 & 7,43 & 8,58 & 1,34 & 1,32 & 0,72 & 6,58 & 7,43 \\
\hline
\end{tabular}

TABLEAU 4 - TABLE 4

Perméabilité et sélectivité expérimentales

Experimental permeability and selectivity

\begin{tabular}{|c|c|c|c|c|c|c|c|c|}
\hline Essai & $\mathbf{J}^{\mathbf{o}_{T}}$ & $\mathrm{Jf}_{T}$ & $\Delta \pi^{\circ}$ & $\Delta \pi^{f}$ & $\mathbf{k}^{\mathrm{o}_{T}}$ & $\mathrm{k}^{\mathbf{f}_{\mathrm{T}}}$ & $\mathrm{TR}^{\circ}$ & $\mathrm{TR}^{\dagger}$ \\
\hline $\mathrm{n}^{\circ}$ & $1 / \mathrm{j} \mathrm{m}^{2}$ & $1 / \mathrm{j} \mathrm{m}^{2}$ & bar & bar & $\underset{\text { bar }}{1 / \mathrm{j} \mathrm{m}^{2}}$ & $\underset{\text { bar }}{1 / \mathrm{j} \mathrm{m}^{2}}$ & $\%$ & $\%$ \\
\hline 1 & 106 & 19 & 20,0 & 20,0 & 2,65 & 0,47 & 73 & 55 \\
\hline 2 & 50 & 26 & 19,0 & 23,5 & 1,22 & 0,71 & 93 & 83 \\
\hline 3 & 119 & 48 & 19,5 & 26,0 & 2,94 & 1,41 & 83 & 66 \\
\hline
\end{tabular}

cours du temps, ainsi que l'indique le tableau 4. Il lui est associé une baisse des coefficients de performance $\mathbf{k}_{\mathrm{T}}$, qui ne saurait être attribuée à un accroissement de la pression osmotique $\pi$ de l'alcool, concomitant à l'augmentation de la concentration en éthanol. En effet, la pression effective $\Delta \mathrm{P}-\Delta \pi$, calculée en ne considérant que l'éthanol, décroît au maximum de $15 \%$ (essai 3) alors que le coefficient de performance correspondant chute de plus de $50 \%$. En outre, la diminution maximale, supérieure à $80 \%$, est atteinte alors que la pression effective ne varie pas (essai 1).

Par ailleurs, quand la vitesse de circulation est multipliée par un facteur 2,5, le coefficient de performance initial $\mathrm{k}_{\mathrm{T}}$ ne varie pas (essais 1 et 3 ), tandis que $\mathrm{k}^{\mathrm{f}_{\mathrm{T}}}$ est triplé. 
Il est donc logique d'attribuer la diminution du coefficient de performance au cours du temps, à la seule augmentation de la résistance au transfert du matériau membraneux par suite d'un encrassement, provoqué par une modification des propriétés superficielles ou par la formation d'un dépôt.

\section{LE BILAN ENERGETIQUE EXPERIMENTAL}

Dans un procédé à membranes, l'apport d'énergie sert à vaincre les résistances au transfert de matière au travers du système membranaire : membrane et couches de polarisation. En régime stationnaire, l'énergie $\mathrm{E}$ fournie au système peut être répartie entre deux grandeurs additives $\mathrm{E}_{\mathrm{p}}$ et $\mathrm{E}_{\mathrm{c}}$, compte tenu de l'incompressibilité des fluides :

$$
\mathrm{E}=\mathrm{E}_{\mathrm{p}}+\mathrm{E}_{\mathrm{c}}
$$

La première, $E_{p}$ est utilisée pour faire passer le volume $V_{p}$ de perméat au travers du système membranaire, en appliquant une pression relative $\Delta \mathrm{P}$.

$$
\mathrm{E}_{\mathrm{p}}=\mathrm{V}_{\mathrm{p}} \cdot \Delta \mathrm{P}
$$

En exprimant le volume du perméat en fonction de la densité du flux transmembranaire $\mathrm{J}_{\mathrm{T}}$, de la durée de l'opération $\mathrm{t}$ et de l'aire utile A de la membrane.

$$
\mathrm{V}_{\mathrm{p}}=\mathrm{A} \cdot \mathrm{J}_{\mathrm{T}} \mathrm{t}
$$

Cette énergie de passage revêt la forme :

$$
\mathrm{E}_{\mathrm{p}}=\mathrm{A} \cdot \mathrm{J}_{\mathrm{T}} \cdot \mathrm{t} \cdot \Delta \mathrm{P}
$$

Notons que par ailleurs la densité du flux s'écrit :

$$
\mathrm{J}_{\mathrm{T}}=\mathrm{k}_{\mathrm{T}} \cdot(\Delta \mathrm{P}-\Delta \pi) \quad(4 \mathrm{bis})
$$

La seconde, $E_{c}$, est mise en œuvre pour diminuer la résistance des couches de polarisation, en l'occurrence dans un module d'osmose inverse, celle située du seul côté du rétentat. Cette réduction de la résistance est étroitement liée à la vitesse de circulation dans le module, autrement dit, au débit $\mathrm{V}$ du rétentat qui traverse le module. Compte tenu de la présence d'une vanne de détente qui assure le maintien de la pression relative $\Delta \mathrm{P}$ par raport à la pression atmosphérique, pendant la durée $\mathrm{t}$ de l'opération :

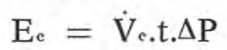

Ainsi, l'énergie fournie pour la séparation est proportionnelle à la durée de l'opération et à la pression relative :

$$
\mathrm{E}=\left(\text { A. } \mathrm{J}_{\mathrm{T}}+\dot{\mathrm{V}}_{\mathrm{c}}\right) \cdot \mathrm{t} \cdot \Delta \mathrm{P}
$$

Des deux termes entre parenthèses, le second est prépondérant, comme le montre le tableau 5. Par conséquent, avec une erreur infé- 
rieure à $0,3 \%$ il est possible d'admettre que toute l'énergie fournie ne sert qu'à réduire la résistance des couches de polarisation :

$$
\mathrm{E} \simeq \mathrm{E}_{\mathrm{e}} \quad(7)
$$

L'obtention d'une vitesse de circulation donnée dans le module, donc d'un débit $\dot{V}_{c}$, est obtenu en effectuant un recyclage permanent du rétentat de volume initial $\mathrm{V}_{\mathrm{R}}$. Le taux de recyclage est alors défini par la relation :

$$
\rho_{\mathrm{cR}}=\frac{\dot{\mathrm{V}}_{\mathrm{e} . \mathrm{t}}}{\mathrm{V}_{\mathrm{R}}^{\mathrm{o}_{\mathrm{R}}}}
$$

Dès lors, l'énergie de circulation s'exprime par la relation :

$$
\mathrm{E}_{\mathrm{c}}=\rho_{\mathrm{cR}} \mathrm{V}_{\mathrm{R}}^{\mathrm{o}} \Delta \mathrm{P}
$$

Pour pouvoir comparer ce coût énergétique à celui d'autres procédés, la dépense d'énergie de circulation $\mathrm{E}_{1}$ a été calculée pour $1 \mathrm{~m}^{3}$ de piquette à traiter. L'ensemble de ces valeurs est rassemblé dans le tableau 5 .

TABLEAU 5 - TABLE 5

Contributions expérimentales à la consommation d'énergie Experimental energy consumptions

\begin{tabular}{c|c|c|c|c|c|c}
\hline \multirow{2}{*}{ Essai } & $10 .{ }^{6} \mathrm{~J}_{\mathrm{T}}$ & $10 .{ }^{9} \mathrm{AJ}_{\mathrm{T}}$ & $10 .{ }^{\circ} \mathrm{V}_{\mathrm{C}}$ & $\rho \mathrm{CR}$ & $\mathrm{E}_{\mathrm{C}}$ & $\mathrm{E}_{1}$ \\
\cline { 2 - 4 } $\mathrm{n}^{\circ}$ & $\mathrm{m}^{3} / \mathrm{m}^{2} \mathrm{~s}$ & $\mathrm{~m}^{3} / \mathrm{s}$ & $\mathrm{m}^{3} / \mathrm{s}$ & - & $\mathrm{M} . \mathrm{J}$ & $\mathrm{MJ} / \mathrm{m}^{3}$ \\
1 & 1,23 & 36,8 & 7,4 & 190 & 11,42 & 1149 \\
2 & 0,58 & 17,3 & 19,3 & 662 & 59,63 & 3975 \\
3 & 1,38 & 41,3 & 19,3 & 338 & 30,44 & 2029 \\
\hline
\end{tabular}

\section{COMPARAISON AVEC LES AUTRES PROCEDES}

La comparaison de la consommation d'énergie peut être faite avec la distillation, soit simple, soit avec recompression mécanique des vapeurs. Cependant, ainsi qu'il a été dit, l'osmose inverse ne peut servir qu'à enrichir une solution alcoolique avant tout autre procédé, et non à effectuer la totalité de la concentration. Aussi, il n'est possible que de déterminer l'économie d'énergie que procure une telle préconcentration. 
En ce qui concerne la distillation simple, les résultats pratiques permettent de calculer le gain d'énergie provoqué par une augmentation du titre d'une solution alcoolique à l'entrée de la colonne. En effet, dans le cas d'une distillation sous pression atmosphérique, la dépense, exprimée en $\mathrm{kg}$ de vapeur par hectolitre d'alcool pour obtenir un distillat à $96 \%$ à partir d'une solution hydroalcoolique de titre donné, est connue (Revuz, 1981). A partir de ces valeurs, l'économie d'énergie par accroissement de la teneur initiale en alcool peut être déterminée en supposant que la vapeur à $120^{\circ} \mathrm{C}$ a un contenu énergétique de $2705 \mathrm{~kJ} / \mathrm{kg}$. Ces calculs, réunis dans le tableau 6, fournissent la valeur de l'économie d'énergie $E_{2}$ pour $1 \mathrm{~m}^{3}$ de solution hydroalcoolique de titre donné à l'entrée.

La comparaison avec une distillation comportant une recompression mécanique des vapeurs peut s'effectuer suivant deux points de vue.

Dans le premier cas, la recompression porte sur les vapeurs de la distillation atmosphérique évoquée précédemment. En admettant un écart de $10^{\circ} \mathrm{C}$ entre les températures de la colonne et celles du compresseur, et en supposant un rendement énergétique de $60 \%$ pour ce dernier, l'économie d'énergie $E_{3}$ s'obtient en divisant les valeurs précédentes (tab. 6) par 5,5.

Dans le second cas, il est possible d'imaginer que la préconcentration par osmose inverse vient se substituer à une distillation limitée avec recompression des vapeurs qui effectuerait le même enrichis-

\section{TABLEAU 6 - TABLE 6}

Economie d'énergie $\mathrm{E}_{2}$, en $\mathrm{MJ} / \mathrm{m}^{3}$, obtenue en distillant une solution hydroalcoolique préconcentrée à $\mathrm{C}^{f}$, au lieu de $\mathrm{C}^{\circ}(\%$ en volume)

Values of $E_{2}$, energy saved, $M J / m^{3}$, when distilling an aqueous solution of alcohol from $C^{f}$ to $C^{\circ}$ (volume \%)

\begin{tabular}{r|c|c|c|c|c|c}
\hline \multicolumn{1}{c|}{$\mathrm{C}^{\circ}$} & 2 & 3 & 4 & 6 & 8 & 10 \\
\hline 2 & & & & & & \\
\hline 3 & 78,9 & 0 & & & & \\
4 & 140,9 & 93,0 & 0 & & & \\
6 & 208,5 & 194,4 & 135,2 & 0 & & \\
8 & 242,3 & 245,1 & 202,9 & 101,4 & 0 & \\
10 & 264,3 & 278,9 & 248,0 & 169,1 & 90,2 & 0 \\
\hline
\end{tabular}


sement. Les bilans matière en eau et en alcool ont été établis en supposant que la membrane a un taux de rejet de $85 \%$. Les valeurs des concentrations étant ainsi fixées pour les solutions riches et pauvres obtenues, le calcul montre que l'énergie nécessaire est divisée en moyenne par 11,6. Les valeurs exactes du facteur correctif sont rassemblées dans le tableau 7. Pour une telle concentration, l'énergie thermique nécessitée par la chaudière n'est pas connue des praticiens. Cependant, il est possible de retenir avec une bonne approximation, celle qui correspond à l'économie obtenue en accroissant le titre de l'alimentation (tab. 6). C'est sur ces bases que le coût énergétique $E_{4}$ pour $1 \mathrm{~m}^{3}$ de solution hydroalcoolique entrante a été calculé : les résultats sont regroupés dans le tableau 7 .

\section{TABLEAU $7-T A B L E 7$}

Dépense d'énergie $E_{4}$, en $M J / \mathrm{m}^{3}$, pour amener une solution hydroalcoolique de la teneur $\mathrm{C}^{\circ}$ à $\mathrm{C}^{f}$ par une distillation avec recompression des vapeurs

$V$ alues of $E_{4}$, energy requirement, $M J / m^{3}$, to increase the concentration (volume \%) of an aqueous solution of alcohol from $C^{\circ}$ to $C^{f}$

\begin{tabular}{|c|c|c|c|c|c|c|}
\hline Operateur & 11,44 & 11,54 & 11,54 & 11,65 & 11,70 & 11,75 \\
\hline Cf & 2 & 3 & 4 & 6 & 8 & 10 \\
\hline 2 & 0 & & & & & \\
\hline 3 & 6,8 & 0 & & & & \\
\hline 4 & 12,2 & 8,0 & 0 & & & \\
\hline 6 & 17,9 & 16,7 & 11,6 & 0 & & \\
\hline 8 & 20,7 & 20,9 & 17,3 & 8,7 & 0 & \\
\hline 10 & 22,5 & 23,7 & 21,1 & 14,4 & 7,7 & 0 \\
\hline
\end{tabular}

Les quatre séries de valeurs obtenues pour l'économie d'énergie montrent que $E_{1}>E_{2}>E_{3}>E_{4}$. En admettant que le traitement par osmose inverse permette de doubler le titre d'une piquette à $4 \%$, l'essai 3 montre que la préconcentration, telle qu'elle a été pratiquée, est dix fois plus onéreuse que la distillation simple. La cause doit en être recherchée dans un taux de recirculation trop élevé : ainsi, l'essai 1 avec les mêmes concentrations, mais avec un débit de circu- 
lation moindre, ne nécessite que la moitié de l'énergie précédente. D'une façon générale, le coût énergétique du traitement de $1 \mathrm{~m}^{3}$ de piquette, sous 60 bars, exprimé en MJ est d'après la relation (9) :

$$
\mathrm{E}_{1}=6 . \rho \mathrm{cR}
$$

Les tableaux $8 \mathrm{a}, \mathrm{b}$ et $\mathrm{c}$, indiquent les valeurs que devrait prendre $\rho$ cR pour que $E_{1}$ soit égale à $E_{2}, E_{3}$ ou $E_{4}$. D'une façon générale, les valeurs de $\rho_{\mathrm{cr}}$ devraient être environ 100 fois plus petites que celles utilisées (tab. 5). De plus, ces tableaux permettent de situer le domaine intéressant des concentrations : par exemple $\rho_{\mathrm{cr}}$ doit être inférieur à 2 pour que $E_{1}$ soit au moins égale à la moitié de $E_{4}$.

Toutefois, la valeur optimale semble bien être $\rho_{\mathrm{c} R}=1$, c'est-àdire qu'il faudrait opérer avec un seul passage de la solution. Ceci est tout à fait possible, puisque les expériences réalisées ont montré que $\rho$ er pouvait être réduit sans inconvénient. De plus, le fait d'opérer avec un seul passage devrait réduire les pertes par évaporation.

Dans la relation qui exprime $\rho \mathrm{er}$, la durée $\mathrm{t}$ de l'opération peut être remplacée par le quotient du volume $V_{p}$ du perméat par le débit transmembranaire A.J J $^{T}$ Le volume du perméat s'obtient à partir des bilans matière de l'eau et de l'éthanol en faisant intervenir le taux de rejet observé TR et le facteur de concentration FC.

\section{TABLEAU $8 a-T A B L E 8 a$}

Valeurs du taux de recyclage $\rho$ cr pour que la dépense d'énergie $E_{1}$ due à l'osmose inverse soit égale à l'énergie $\mathrm{E}_{2}, \mathrm{E}_{3}, \mathrm{E}_{4}$ pour amener une solution hydroalcoolique de la teneur $\mathrm{C}^{\circ}$ à $\mathrm{C}^{\mathrm{f}}$ (\% en volume)

Values of the recirculation rate por to obtain the same energy consumption in reverse osmosis $\left(E_{1}\right)$ as in distillation $\left(E_{2}, E_{3}, E_{4}\right)$ when concentring an aqueous solution of alcohol from $C^{\circ}$ to $C^{f}$

\begin{tabular}{|c|c|c|c|c|c|c|}
\hline $\mathrm{Cf}$ & $\mathrm{C}^{\circ}$ & 2 & 3 & 4 & 6 & 8 \\
\hline 3 & & 13,1 & & & & \\
\hline 4 & & 23,5 & 15,5 & & & \\
\hline 6 & & 34,8 & 32,4 & 22,5 & & \\
\hline 8 & & 40,4 & 40,8 & 33,8 & 16,9 & \\
\hline 10 & & 44,0 & 46,5 & 41,3 & 28,2 & 15,0 \\
\hline
\end{tabular}

8a : $\mathrm{E}_{1}=\mathrm{E}_{2}$ (distillation simple).

$8 a: E_{1}=E_{2}$ (conventional distillation). 
TABLEAU $8 b-T A B L E 8 b$

\begin{tabular}{|c|c|c|c|c|c|}
\hline $\mathrm{Cf}$ & 2 & 3 & 4 & 6 & 8 \\
\hline 3 & 2,4 & & & & \\
\hline 4 & 4,3 & 2,8 & & & \\
\hline 6 & 6,3 & 5,9 & 4,1 & & \\
\hline 8 & 7,3 & 7,4 & 6,1 & 3,1 & \\
\hline 10 & 8,0 & 8,4 & 7,5 & 5,1 & 2,7 \\
\hline
\end{tabular}

$8 \mathrm{~b}: \mathrm{E}_{1}=\mathrm{E}_{3}$ (distillation de $\mathrm{C}^{\mathrm{f}}$ à $96 \%$, avec recompression des vapeurs). $8 b: E_{1}=E_{3}$ (distillation from $C^{f}$ to $96 \%$ with vapor recompression).

TABLEAU $8 c-T A B L E 8 c$

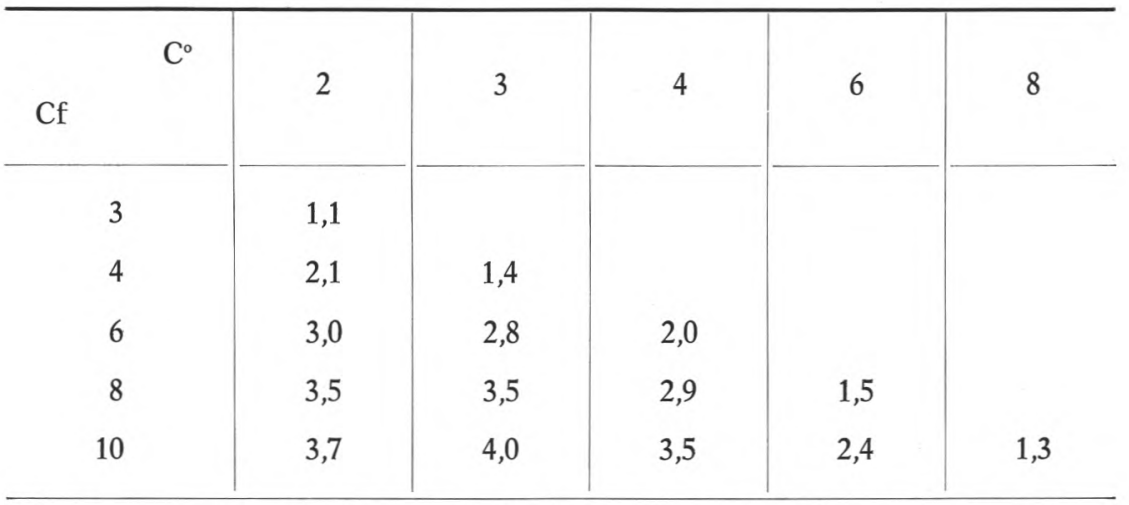

$8 \mathrm{c}: \mathrm{E}_{1}=\mathrm{E}_{4}$ (distillation de $\mathrm{C}^{\circ}$ à $\mathrm{C}^{\mathrm{f}}$ avec recompression des vapeurs).

$8 c: E_{1}=E_{4}$ (distillation from $C^{\circ}$ to $C^{f}$ with vapor recompression).

$$
\mathrm{V}_{\mathrm{p}}=\mathrm{V}_{\mathrm{R}}^{\mathrm{o}} \cdot \frac{\mathrm{FC}-1}{\mathrm{TR} \cdot \mathrm{FC}}
$$

Finalement, le taux de recyclage prend la forme :

$$
\rho_{\mathrm{e} R}=\frac{\mathrm{V}_{\mathrm{e}}}{\mathrm{A}_{\mathrm{J}} \mathrm{J}_{\mathrm{T}}} \frac{\mathrm{FC}-1}{\mathrm{TR} \cdot \mathrm{FC}}
$$


L'expression de l'énergie de recyclage $E_{\mathrm{c}}$ met alors en évidence, une fois les objectifs $V_{\mathrm{R}}^{\circ}$ et FC fixés, les conditions opératoires par $\dot{V}_{\mathrm{e}}$, les caractéristiques des membranes par TR et $\mathrm{k}_{\mathrm{T}}$ et les dimensions du module par sa surface A :

$$
\mathrm{E}_{\mathrm{c}}=\frac{\mathrm{FC}-1}{\mathrm{FC}} \frac{\mathrm{V}_{\mathrm{R}}}{\mathrm{A}} \frac{\dot{\mathrm{V}}_{\mathrm{e}}}{\mathrm{TR}} \frac{1}{\mathrm{k}_{\mathrm{T}}}
$$

Habituellement TR et $\mathrm{k}_{\mathrm{r}}$ varient en sens inverse. Dans le cas où il existe une relation linéaire de la forme :

$$
\mathrm{k}_{\mathrm{T}}=\mathrm{a}-\mathrm{b} . \mathrm{TR}
$$

La valeur maximale du produit est $\mathrm{a}^{2} / 4 \mathrm{~b}$; le taux de rejet observé vaut alors $a / 2 b$ et le coefficient de performance $a / 2$. Par exemple, d'après les valeurs de la figure 3 , pour les membranes $\mathrm{X}$, le maximum donne une valeur de $\mathrm{k}_{\mathrm{w}}$ proche de $16 \mathrm{l} / \mathrm{j} \cdot \mathrm{m}^{2}$.bar, TR étant voisin de $50 \%$. Mais alors FC ne saurait être supérieur à 2, ce qui, finalement conduit à un volume de rétentat négligeable; or ce n'est pas le but recherché.

Une fois TR et $\mathrm{k}_{\text {r }}$ fixés, l'énergie de circulation ne saurait être réduite qu'en augmentant l'aire d'échange $\mathrm{A}$. Cet accroissement de la surface de transfert peut être obtenu soit en multipliant le nombre de motifs élémentaires s'il s'agit d'un module à plaques, ou d'un module tubulaire, soit en utilisant un autre module, par exemple équipé de fibres creuses.

Cependant, l'augmentation de l'aire ne saurait se poursuivre indéfiniment, car la vitesse tangentielle tomberait à une valeur telle, que la résistance des couches de polarisation deviendrait excessive.

\section{CONCLUSION}

Les essais de préconcentration en alcool des piquettes par osmose inverse avec les membranes disponibles actuellement montrent que l'opération est techniquement possible. Toutefois, elle ne saurait conduire à une économie de la dépense d'énergie que si le génie du procédé est parfaitement maîtrisé.

$\mathrm{Au}$ vu des expériences réalisées, il est nécessaire de recycler le moins possible les piquettes, le mieux étant d'opérer avec un seul passage. En outre, même dans ce cas, l'énergie de transfert est moindre que celle de circulation. Pour une concentration déterminée, cette dernière peut être réduite si le produit TR.k est $_{\text {accru. Compte }}$ tenu de la perméabilité et de la sélectivité des membranes disponibles, il est donc indispensable d'avoir recours à des modules présentant une grande surface de transfert. 
Néanmoins, il est patent que la confection de membranes plus performantes, est aussi un moyen à utiliser.

De plus, cette comparaison effectuée du seul point de vue de l'énergie, n'a pas pris en compte les dimensions des appareillages, qui reposent sur la valeur des débits de piquette à traiter. Il est bien évident que si l'osmose inverse peut, par son caractère modulaire, s'adapter à toutes les situations, la distillation simple, et surtout la distillation avec recompression des vapeurs, nécessitent un débit minimum non négligeable.

Cependant, les optimums technico-économiques ne pourraient être déterminés que dans la mesure où un certain nombre d'essais supplémentaires auront fourni les éléments de calcul.

\section{NOTATIONS}

Alphabet latin

A : aire utile de membrane $\left(\mathrm{m}^{2}\right)$.

$\mathrm{a}, \mathrm{b}$, : constantes $\left(\mathrm{kg} / \mathrm{j} \mathrm{\textrm {m } ^ { 2 }}\right.$ bar).

C : concentration ( $\%$ en volume).

$\mathrm{C}_{\mathrm{P}} \quad$ : concentration du perméat ( $\%$ en volume).

$\mathrm{C}_{\mathrm{R}}$ : concentration du rétentat ( $\%$ en volume).

E : énergie fournie (joule).

$\mathrm{E}_{\mathrm{C}} \quad$ : énergie de circulation (joule).

$E_{P} \quad$ : énergie de transfert (joule).

$\mathrm{E}_{1}$ : dépense d'énergie en O.I. $\left(\mathrm{J} / \mathrm{m}^{3}\right)$.

$\mathrm{E}_{2}$ : économie d'énergie pour la distillation simple $\left(\mathrm{J} / \mathrm{m}^{3}\right)$.

$\mathrm{E}_{3}$ : économie d'énergie pour la distillation avec recompression des vapeurs $\left(\mathrm{J} / \mathrm{m}^{3}\right)$.

$\mathrm{E}_{4}$ : dépense d'énergie pour la distillation avec recompression des vapeurs $\left(\mathrm{J} / \mathrm{m}^{3}\right)$.

FC : facteur de concentration.

$\mathrm{J}_{\mathrm{T}}$ : densité du flux transmembranaire $\left(\mathrm{kg} / \mathrm{j} \mathrm{m} \mathrm{m}^{2}\right.$ ou $\left.1 / \mathrm{j} \mathrm{m}^{2}\right)$.

$\mathbf{k}_{\mathrm{T}}$ : coefficient de performance $\left(\mathrm{kg} / \mathrm{j} \mathrm{\textrm {m } ^ { 2 }}\right.$ bar).

O.I. : osmose inverse.

$\mathrm{P}_{\mathrm{C}}$ : pression de colmatage.

$\mathrm{T}$ : température (K).

t : durée (h).

TRG : toux de rejet global.

TR taux de rejet observé.

$\mathrm{u}$ : vitesse de circulation $(\mathrm{cm} / \mathrm{s})$.

$\mathrm{V}_{\mathrm{P}}$ : volume du perméat $\left(\mathrm{m}^{3}\right)$.

$\mathrm{V}^{\mathrm{o}}{ }_{\mathrm{R}}$ : volume initial de solution (rétentat) $\left(\mathrm{m}^{3}\right)$.

$\dot{\mathrm{V}}_{\mathrm{C}} \quad$ : débit de circulation du rétentat $\left(\mathrm{m}^{3} / \mathrm{s}\right)$. 
Alphabet grec

$\Delta \mathbf{P}$ : pression relative (bar, atm).

$\Delta \pi \quad$ : différence de pression osmotique (bar).

$\rho \mathrm{CR}:$ taux de recyclage du rétentat.

Indices et exposants

C : circulation.

F : fuite.

f : final.

o : initial.

P : péerméat ou osmosat.

$\mathrm{R}$ : rétentat ou concentrat.

$\mathrm{T}$ : transmembranaire.

\section{Remerciements}

Nous tenons à remercier $M$. Dick, de l'IRCHA, qui nous a obligeamment fourni les échantillons de membrane, et $M$. Barale qui a bien voulu nous accueillir dans les locaux du CEPICA.

\section{Bibliographie}

CABRIT (M.) (1982). - Relèvement par osmose inverse du degré alcoométrique des piquettes à distiller. Mémoire de fin d'études, E.S.A.P.

GREGoR (H.), JeFFrIE (S.) (1979). - Ethanolic fuels from renewable resources in the solar age. N.T.I.S., PB 295645.

Mafart (D.), 1981). - Coût énergétique des opérations d'élimination d'eau. Edité par Technique et Documentation, Paris.

Menta (G.) (1982). - Comparison of membrane process with distillation for alcohol water separation. J. Memb. Science, 12 (1), 1-26.

Moutounet (M.), LuRton (L.), Audinos (R.) (1981). - Electrodialyse appliquée aux moûts de raisin. Colloque Société Française de Filtration, Montpellier.

Revuz (B.) (1981). - Procédés d'extraction de l'éthanol et fermentation extractive. Colloque Société Française de Microbiologie, Reims: 